\title{
Pseudo-random Single Photon Counting for Space-borne Atmospheric Sensing Applications
}

\author{
Xiao Ai \\ Richard William Nock \\ Naim Dahnoun \\ John Rarity
}

\author{
Antonio Consoli \\ Ignacio Esquivias \\ Universidad Politécnica \\ de Madrid
}

\author{
Mathieu Quatrevalet \\ Gerhard Ehret
}

\begin{abstract}
The ability to accurately observe the Earth's carbon cycles from space gives scientists an important tool to analyze climate change. Current space-borne Integrated-Path Differential Absorption (IPDA) lidar concepts have the potential to meet this need. They are mainly based on the pulsed time-offlight principle, in which two high energy pulses of different wavelengths interrogate the atmosphere for its transmission properties and are backscattered by the ground. In this paper, feasibility study results of a Pseudo-Random Single Photon Counting (PRSPC) IPDA lidar are reported. The proposed approach replaces the high energy pulsed source (e.g. a solidstate laser), with a semiconductor laser in CW operation with a similar average power of a few Watts, benefiting from better efficiency and reliability. The auto-correlation property of Pseudo-Random Binary Sequence (PRBS) and temporal shifting of the codes can be utilized to transmit both wavelengths simultaneously, avoiding the beam misalignment problem experienced by pulsed techniques. The envelope signal to noise ratio has been analyzed, and various system parameters have been selected. By restricting the telescopes field-of-view, the dominant noise source of ambient light can be suppressed, and in addition with a low noise single photon counting detector, a retrieval precision of $1.5 \mathrm{ppm}$ over $50 \mathrm{~km}$ along-track averaging could be attained. We also describe preliminary experimental results involving a negative feedback Indium Gallium Arsenide (InGaAs) single photon avalanche photodiode and a low power Distributed Feedback laser diode modulated with PRBS driven acoustic optical modulator. The results demonstrate that higher detector saturation count rates will be needed for use in future spacebourne missions but measurement linearity and precision should meet the stringent requirements set out by future Earthobserving missions.
\end{abstract}

\section{TABLE OF CONTENTS}

1 InTRODUCTION...........................

2 PULSED IPDA LIDAR $\ldots \ldots \ldots \ldots, \ldots, \ldots, \ldots, \ldots, 2$

3 Proposed PRN Modulation .............. 3

4 Preliminary ExPERIMENTAL Results....... 6

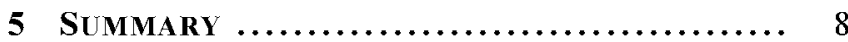

ACKNOWLEDGMENTS ...................... 8

REFERENCES $\ldots \ldots \ldots \ldots \ldots \ldots \ldots \ldots \ldots \ldots \ldots, 8$

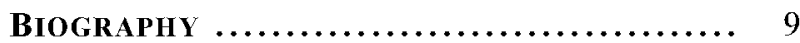

\section{INTRODUCTION}

$\mathrm{CO}_{2}$ is a major greenhouse gas contributing towards global warming and climate change. Therefore, there is an urgent need to monitor the exchanges of carbon between the atmosphere and both the ocean and the land surfaces. In the so-called top-down approach, the surface fluxes of $\mathrm{CO}_{2}$ are inverted from the observed spatial and temporal concentration gradients. Currently, the concentrations of $\mathrm{CO}_{2}$ are mainly measured in-situ at a number of surface stations that are unevenly distributed over the Earth. Satellite missions have the potential to provide a denser and better distributed set of observations to complement this network. Thus, developing satellite techniques for $\mathrm{CO}_{2}$ observation is important to improve our knowledge of carbon sources and sinks. To address the needs for $\mathrm{CO}_{2}$ monitoring, a variety of studies, developments and experimental demonstrations are currently being carried out. In particular, the IPDA lidar technique [1], which sets the framework of this study, uses hard target reflection in the near IR to measure the column averaged dry air $\mathrm{CO}_{2}$ mixing ratio. The potential precision of this approach has been found to be adequate for fulfilling observational requirements.

The primary advantage of a lidar mission is that it does not require the sun as a light source, and can therefore provide both day and night, all-seasons and all latitude measurements and thus will provide an increase in the number of observations by a factor of two to three compared to passive missions. Furthermore, active techniques are less sensitive to the presence of aerosols.

The pulsed IPDA lidar (Figure 1), such as the $\mathrm{CO}_{2}$ and $\mathrm{CH}_{4}$ Atmospheric Remote Monitoring-Flugzeug (CHÂRMF) developed by the by the Deutsches Zentrum für Luft-und Raumfahrt (DLR) [2] estimates the column concentration of greenhouse gases in the atmosphere by looking at the backscattered pulse echoes at the end of the optical path, which is either the cloud top or the Earth's surface. The term "differential' applies to a pair of lasers with two slightly different wavelengths: one (on-line) is near the center of a $\mathrm{CO}_{2}$ absorption line; the other (off-line) is set close to but off the same line. Both wavelengths are close enough to exhibit almost identical aerosol attenuations, but will exhibit different $\mathrm{CO}_{2}$ absorptions. Hence, the attenuation from $\mathrm{CO}_{2}$ molecules can be calculated by the power ratio of the backscattered signals at the end of the optical path and can be converted into a column-averaged mixing ratio thanks to the knowledge of the path length from the round-trip time delay. Because both channels share the same detector, to avoid ambiguity of the returned on-line and off-line pulse pairs, the temporal separation between these two pulses must be set to more than the round-trip time from the top of the atmosphere to ground. This results in a misalignment of the footprints of the pulse pairs which deteriorates detection accuracy due to the associated ground reflectance and alti- 


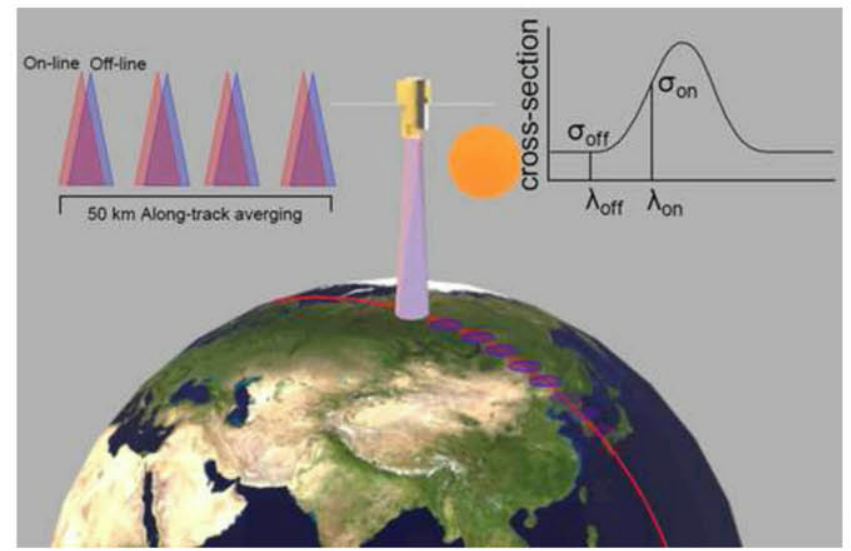

Figure 1. Space-borne pulsed IPDA lidar instrument: (left) shows the on-line and off-line beams sequentially transmitted and along track averaging length of $50 \mathrm{~km}$; (middle) illustrates the space-borne instrument in operation; (right) plots a typical $\mathrm{CO}_{2}$ absorption cross-section curve, with the on-line and off-line wavelengths denoted.

tude variations, as shown in Figure 2(a). The CW Laser Absorption Spectroscopy (LAS) [3] is another approach to IPDA lidar, which modulates the on-line and off-line lasers with sine-waves of different frequencies. The use of lock-in detection at the receiver allows for the received power from the on and off-lines to be discriminated. However, due to the periodic modulation, the ground reflection is interfered with back-scattered signals from volumetric targets such as cirrus clouds and dust, as illustrated in Figure 2(b).

Pseudo-Random Noise (PRN) or Random Modulation Continous Wave (RM-CW) lidar with analogue detection was first applied in [4] for aerosol detection the in $\mathrm{km}$ range. It is capable of obtaining range gated back-scattering information as obtained from pulsed techniques. It has been applied in Differential Absorption LIDAR (DIAL) measurements [5], and Bristol University has enhanced its distance precision in [6]. In PRN lidar, a PRBS is transmitted, and the received signal is correlated with the original PRBS code giving range resolved response with non-ambiguous range as determined by the number of PRBS bits (repetition rate) which can be extended further than the depth of atmosphere. Overall,

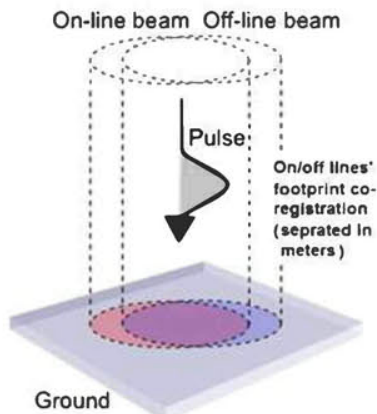

(a)

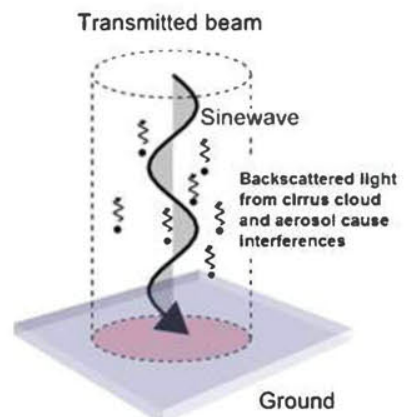

(b)
Figure 2. Drawbacks of existing IPDA techniques: (a) the pulsed approach suffers from on/off line lasers' footprint misalignments; (b) using the sine-wave modulated CW approach leads to the signal being mixed with backscattered sine-wave from the volumetric targets. the average power of a PRN modulated system would be increased beyond that of a typical pulsed system which will be limited by damage effects and non-linear frequency drift at high peak powers. Hence although there is a slight signal to noise (SNR) reduction in PRN modulated systems due to ambient shot noise [7], the increased numbers of photons sent should amply compensate for this deficiency.

The auto-correlation property of PRBS and temporal shifting of the codes can be utilized to transmit both wavelengths simultaneously, thereby avoid the beam misalignment problem. Furthermore, due to the extended non-ambiguous distance, equals to the total PRBS code length, the returns can be binned into different range gates with the distance resolution related to the PRBS bit time (chip time). Its potential to be used in IPDA lidar has also recently been recognized by [8], where PRBS modulation has been adapted to LAS systems. However due to the ac-coupled analogue receiver, a carrier frequency needs to be mixed with the applied PRBS code, resulting in distortions to the auto-correlation, and the applied linear mode detector adds significant detector noise.

The availability of suitable laser sources is one of the main challenges in future space missions for atmospheric remote sensing. Typical laser sources currently used in DIAL systems are solid state lasers working in the pulsed regime, emitting ns pulses with high energy at low to medium repetition rate (typical values are $10 \mathrm{~ns}$ to $50 \mathrm{~ns}, 10 \mathrm{~mJ}$ to $50 \mathrm{~mJ}, 50 \mathrm{~Hz}$ to $200 \mathrm{~Hz}$ ) [9], [10]. Although these laser systems have demonstrated the high average power, high laser beam quality and frequency stability required by the application, it is at the expense of a bulky system with low wall plug efficiency, which is a main concern for space-borne applications. Numata et al. [11] have investigated hybrid Master Oscillator Power Amplifierss (MOPAs) combining a Distributed Feedback (DFB) semiconductor laser as seed laser and Erbium Doped Fiber Amplifiers working in pulsed conditions in the context of the ASENDS NASA mission. However, the use of active optical fibers in space applications requires specific attention to radiation shielding, orbit and the duration of the flight, since it is known that fiber amplifier materials have low radiation hardness [12]. All-semiconductor laser sources are suitable candidates for space-borne atmospheric sensing applications, due to their compactness, high efficiency, reliability, and radiation hardness. They cannot produce the high energy pulses needed in standard pulsed IPDA lidar, but they are well suited to PRN systems. The European Project BRITESPACE [13] is developing an allsemiconductor laser source able to fulfill the requirements of future $\mathrm{CO}_{2}$ monitoring space missions, based on an integrated multi-section MOPA.

In the frame of BRITESPACE project, we report a feasibility study of a new type of IPDA lidar for space-borne remote sensing applications. The proposed system applies the PRSPC [14], [15] technique and uses a high-gain, low noise DC-coupled Single Photon Avalanche Photodiode (SPAD). It also incorporates two high brightness semiconductor laser sources, one for each sounding frequency, emitting near $1.572 \mu \mathrm{m}$. Preliminarily experimental results have proven its feasibility in terms of precision and linearity.

\section{PULSED IPDA LIDAR}

In this section, a pulsed IPDA lidar (CHARM-F) is described following the guidelines set out by [2], allowing for a direct comparison with the proposed PRSPC system. In a pulsed 
IPDA lidar, a pair of laser pulses is transmitted sequentially with pulse energies of $E_{o n}^{t}$ and $E_{o f f}^{t}$. The ground echoes can be calculated via the lidar equation as shown in Eq. (1), in which $\eta_{o}$ denotes the optical efficiency; $\alpha_{s}$ is the surface reflectivity of the ground (Lambertian) surface; $A_{r}$ is the receiving telescope area; $T_{\text {on }, \text { of } f}$ denotes the two way atmospheric transmittance and is related to the total column optical depth $O D_{o n, o f f}$ as shown in Eq. (2). The total column optical depth includes a wavelength-independent term (from aerosols and thin clouds), $O D_{0}$ and the $\mathrm{CO}_{2}$ optical depth, the latter being further related to the altitude $r$ dependent effective absorption cross-section $\sigma(r)$ and the number density $n_{c o 2}(r)$ as shown in Eq. (3), where $R$ represents the distance from the orbit altitude to ground.

$$
\begin{gathered}
E_{\text {on }, o f f}^{r}=E_{\text {on }, o f f}^{t} \cdot \eta_{o} \cdot \alpha_{s} \cdot T_{o n} \cdot \frac{A_{r}}{R^{2}}, \\
T_{\text {on }, \text { off }}=\exp \left(-2 \cdot O D_{\text {on }, o f f}\right), \\
O D_{o n, o f f}=\int_{0}^{R} \sigma_{o n, o f f}(r) \cdot n_{c o 2}(r) d r+O D_{0} .
\end{gathered}
$$

The differential absorption optical depth (DAOD) is defined as $D A O D=O D_{\text {on }}-O D_{\text {off }}$. It quantifies the molecular absorption by $\mathrm{CO}_{2}$ along the path between the scattering surface and the instrument and can be calculated according to Eq. (1) and Eq. (3) as shown in Eq. (4). Hence, a link between the received on-line echo $E_{\text {on }}^{r}$, off-line echo $E_{\text {off }}^{r}$ and the $\mathrm{CO}_{2}$ number density of the probed column has been established. Note that, herein we refer the transmitted on/off lines as the transmitted channels, and the received signals of the on/off lines as received channels.

$$
\begin{aligned}
D A O D & =\frac{1}{2} \ln \left(\frac{E_{o f f}^{r} \cdot E_{o n}^{t}}{E_{o n}^{r} \cdot E_{o f f}^{t}}\right) \\
& =\int_{0}^{R}\left(\sigma_{o n}(r)-\sigma_{o f f}(r)\right) \cdot n_{c o 2}(r) d r .
\end{aligned}
$$

The dry-air mixing ratio of $\mathrm{CO}_{2} X_{\mathrm{co} 2}$ is related by the number density $n_{\mathrm{CO} 2}(r)$ and the number density of the dry air $n_{\text {air }}(r)$ by $X_{c o 2}=n_{c o 2}(r) / n_{a i r}(r)$. Assuming the $X_{c o 2}$ is invariant with altitude, the column weighted mixing ratio of $\mathrm{CO}_{2}$ can be represented by Eq. (5), where the altitude dependent weighting function $W F(r)$ removes bias due to temperature, atmospheric pressure along the path, and is typically derived from analysis of Numerical Weather Prediction (NWP) modeled data. $W F(r)$ can be expanded as shown in Eq. (6), according to the relationship between the temperature $T_{e}(r)$, pressure $P_{a}(r)$ and number density of dry air $N_{\text {air }}(r)$ via the $D A O D$. Note that, because the laser source would not be purely monochromatic (spectrum broadening due to modulation and finite laser line-width), $\sigma_{\text {on }, o f f}(r)$ used in Eq. (6) should be the effective absorption cross-section, which considers these factors. $A_{v}$ is the Avogadro's number and $R_{c}$ denotes the gas constant.

$$
\begin{aligned}
X_{c o 2} & =\frac{\int_{0}^{R}\left(\sigma_{o n}(r)-\sigma_{o f f}(r)\right) \cdot n_{c o 2}(r) d r}{\int_{0}^{R}\left(\sigma_{o n}(r)-\sigma_{o f f}(r)\right) \cdot n_{a i r}(r) d r} \\
& =\frac{D A O D}{\int_{0}^{R} W F(r) d r}, \\
W F(r) & =\frac{A_{v}}{R_{c}} \cdot \frac{P_{a}(r)}{T_{e}(r)} \cdot\left(\sigma_{o n}(r)-\sigma_{o f f}(r)\right) .
\end{aligned}
$$

Consequently, the $X_{\mathrm{CO} 2}$ can be calculated from the following information: (a) the measured DAOD by the energy ratio of the transmitted and received on/off line channels, as shown in Eq. (4); (b) the NWP modeled data (pressure, temperature etc.), and the effective absorption cross-section; and (c) the orbit altitude to ground distance $R$ (calculated by the roundtrip time delay of received pulses). Furthermore, by proper selection of the sounding on and off-line wavelengths, possible measurement biases due to atmospheric water vapor can be significantly reduced [16].

It is expected that averaging through a $50 \mathrm{~km}$ ground track will be required to achieve the desired detection precision. In the case of CHARM-F, the averaging is carried out on $X_{\mathrm{co} 2}$ measurements obtained from $M$ pairs of on-line and off-line returns. With a typical pulse pair repletion rate of $50 \mathrm{~Hz}$ and satellite ground speed of $7 \mathrm{~km} \mathrm{~s}^{-1}$, the number of such pulse-pairs averaged is $M=350$ for $50 \mathrm{~km}$. This results in the averaged column weighted dry air mixing ratio of $\mathrm{CO}_{2}$, denoted as $\mathrm{XCO} 2$ as:

$$
X C O 2=\frac{1}{M} \sum_{i=1}^{M} X_{c o 2}(i) .
$$

\section{Proposed PRN Modulation}

Pseudo-Random Noise is mathematically intractable. Hence, to describe PRN modulation, the simple model Eq. (1-7) is expanded to include time. The waveform analysis of the pulsed method is then applied to the PRN technique using the autocorrelation property of a random impulse train. In a pulsed system, the transmitted pulse power can be described by:

$$
P_{o n, o f f}^{t}=P_{o n, o f f}^{k} \cdot \delta(t) * G_{p}(t)
$$

where, $*$ denotes convolution; $P_{\text {on, of } f}^{k}$ represents the values of the laser peak powers; $\delta(t)$ is the Dirac function and $G_{p}(t)$ describes the shape of the transmitted laser pulse. For example a square pulse with width $T_{p u l}$ can be described by:

$$
G_{p}(t)= \begin{cases}1 & |t| \leq T_{p u l} \\ 0 & |t|>T_{p u l}\end{cases}
$$

Note that, in Eq. (8) the transmitted power is related to the transmitted energy as $E_{\text {on, off }}^{r}(t)=\int P_{\text {on }, o f f}^{r}(t) d t$.

Light that penetrates through the atmosphere is returned to the detector by volumetric backscattering (aerosol, cirrus clouds 
and dusts) and ground reflection and can be described by $P_{o n, o f f}^{t}(t)$ convolved with the atmosphere response function $G_{a}(t)$ as shown in Eq. (10). For example, assuming for the sake of simplicity the only significant refection is from the ground (Eq. (11)), in which the round-trip time-of-flight of the ground reflection $\tau$ equals to $2 R / c$. However, when considering a more general case where volumetric backscattering is considered, Eq. (8) and (10) combine to Eq. (12).

$$
\begin{gathered}
P_{o n, o f f}^{r}(t)=P_{o n, o f f}^{t}(t) * G_{a}(t), \\
G_{a}(t)=\eta_{0} \cdot \alpha_{s} \cdot T_{o n, o f f} \cdot \frac{A_{r}}{R^{2}} \cdot \delta(t-\tau), \\
P_{o n, o f f}^{r}(t)=P_{o n, o f f}^{k} \cdot \delta(t) * G_{p}(t) * G_{a}(t) .
\end{gathered}
$$

With the received waveform expressed by Eq. (12), the PRN lidar can be derived by expanding the impulse $\delta(t)$ function into Eq. (13) using the auto-correlation property of the random impulse train $I I I_{T}(t)$, with terms expressed by Eq. (14) and Eq. (15), where $a_{k}$ represents a PRBS as a sequence of pseudo-random binary numbers, and $T_{c}$ denotes the period of the impulse train. Note that correlation is represented in Eq. (12) as a convolution with a flipped time axis.

$$
\begin{gathered}
\delta(t)=I I I_{T}(t) *\left(I I I_{T}(-t)-\overline{I I I}_{T}(-t)\right), \\
I I I_{T}(t)=\sum_{k} a_{k} \delta(t-k T), \\
\overline{I I I}_{T}(t)=\sum_{k} \bar{a}_{k} \delta(t-k T) .
\end{gathered}
$$

Subsequently, the received power can be described by substituting Eq. (13) into Eq. (12), resulting in Eq. $(16,17)$, which is the model of the PRN lidar scheme. It should be equivalent to the pulsed scheme in noiseless conditions. For $S_{r}(t)$, with the terms in the square brackets representing a pseudorandom waveform with a peak power of $P_{o n, o f f}^{k}$, which is transmitted through the atmosphere and backscattered, a process described by the response function $G_{a}(t)$. Hence, terms in curly brackets describe the received signal $S_{r}(t)$. Finally, the received signal is correlated with the random impulse train $I I I_{T}(t)$ and the negated impulse train $\overline{I I I}_{T}(t)$.

$$
\begin{gathered}
P_{o n, o f f}^{r}(t)=S_{r}(t) * I I I_{T}(-t)-S_{r}(t) * \overline{I I I}_{T}(-t) . \\
S_{r}(t)=\left\{\left[P_{o n, o f f}^{k} \cdot I I I_{T}(t) * G_{p}(t)\right] * G_{a}(t)\right\} .
\end{gathered}
$$

Up to this point the model lacks consideration of the ambient light power. It can be easily proved that ambient light level does not generate any bias in measurements via Eq. (18), where $C$ can take any value for it to be held true.

$$
\delta(t)=\left(I I I_{T}(t)+C\right) *\left(I I I_{T}(-t)-\overline{I I I}_{T}(-t)\right)
$$

Consequently, when the on/off wavelengths are transmitted sequentially, the proposed PRN system is able to obtain correlation results that are equal to the received powers in the case of a pulsed system. Because the maximum atmospheric depth is known (maximum round-trip time delay of $T_{\max }$ ), a code with repetition period longer than $2 \cdot T_{\max }$ is utilized. Time delaying the modulation code of the offline for $T_{\max }$ in reference to that of the on-line allows for both wavelengths to be transmitted together, and the returns can be received simultaneously using a singular detector and decoded separately. The combined transmitted signal is thus described by Eq. (19). The time delay of a single code for multi-wavelength measurements utilizing the known maximum distance of the returns has been recognized recently in [8].

$$
P_{o n}^{k} \cdot I I I_{T}(t) * G_{p}(t)+P_{o f f}^{k} \cdot I I I_{T}\left(t-T_{\max }\right) * G_{p}(t) .
$$

\section{Derivation of the noise model}

In the previous sub-section, it was demonstrated that the PRN modulation yields equivalent results to the pulsed approach in noiseless conditions. In the following sub-section, the noise performance of the system in space-borne scenarios will be investigated. It has been identified that the major noise contributor of the proposed system will be signal/ambient shot noise and detector dark noise. Similar to previous derivations, the SNR will be first described for the pulsed approach. For the pulsed scheme, the received pulse energy $E_{o n, o f f}^{r}$ is concentrated within a single range gate (main refection from the ground). Signal shot noise is proportional to the square-root of the number of photoelectrons integrated in this range gate, $N_{o n, o f f}=\xi_{e} \cdot E_{o n, o f f}^{r}$, in which $\xi_{e}$ denotes the photon energy to photoelectron number conversion coefficient (see Table 1). Ambient light and detector noise equivalent photoelectron numbers are represented by $N_{a m b}$ and $N_{\text {det }}$ per range gate (bin). Note that, although the detector noise is not technically shot noise, it is conventional to express it as the equivalent shot noise power induced by an ambient photoelectron number in units of noise equivalent power (NEP). The effect of such noise sources will only be experienced in the bin where $N_{\text {on,off }}$ reside. Note that the pulsed approach is not only capable of range gating the returns, but it is also gating the noise that contributes towards the measured return energy. Following the shot-noise statistics, the SNR of the pulsed method can be approximated by:

$$
S N R_{o n, o f f}^{p u l}=\frac{N_{o n, o f f}}{\sqrt{N_{o n, o f f}+N_{a m b}+N_{d e t}}} .
$$

To compare the SNR of the PRN system with respect to the pulsed system, it will be assumed that both systems have the same average transmission power, which yields the same total received signal photoelectron numbers $N_{\text {on, off }}$ after averaging (correlation). A PRN system under such conditions will receive fewer photoelectrons per range gate, as energy is spread over $m$ (number of ' 1 's in the PRBS) range gates, while the received photoelectrons counts per range gate are 
reduced by a factor of $m$. The number of photoelectrons for ambient light $N_{a m b}$ and noise equivalent photoelectrons $N_{\text {det }}$ per gate remain the same as the pulsed system. In the PRN system, the cross-correlation effectively accumulates the photoelectrons numbers for $m$ range gates. Hence, a high level of noise photoelectrons $m \cdot N_{a m b}$ and $m \cdot N_{\text {det }}$ per correlation (integration) time is to be expected. Therefore,

$$
\begin{aligned}
& S N R_{o n, o f f}^{p r n}= \\
& \frac{N_{o n, o f f}}{\sqrt{2} \sqrt{N_{o n, o f f}+N_{o f f, o n}+m \cdot N_{a m b}+m \cdot N_{\text {det }}}} .
\end{aligned}
$$

Note that in Eq. (21), the square-root 2 in the noise term is caused by the subtraction of two correlations described in Eq. (16). Both $N_{\text {on }}$ and $N_{\text {off }}$ are in the noise term (denominator), because both on/off-line wavelengths are transmitting simultaneously during each retrieval.

According to Eq. (19-21), for the precision of the PRN system (Eq. (22)) to approach to the precision of the pulsed system with similar average transmitting power, the ambient light level and detector noise needs to be reduced.

$$
\sigma_{x c o 2}=\frac{X C O 2}{2 \cdot D A O D} \cdot \sqrt{\left(S N R_{o n}^{p r n}\right)^{-2}+\left(S N R_{o f f}^{p r n}\right)^{-2}} .
$$

\section{Ambient and Detector Noise Reduction}

Reduction of the ambient light level and consequent improvement of the SNR can be obtained by adequate optical filtering techniques. NASA has carried out studies on utilizing Fiber Bragg Grating (FBG) filters for narrow band filtering in atmospheric sensing applications [17]. Such filters would yield a $32 \mathrm{pm}$ bandwidth, and stretching or heating allows fine tuning. However, FBGs require a single mode fiber, but the typical spot size that can be achieved in suitable telescopes at focus is typically around $50 \mu \mathrm{m}$, which leads to a low telescope to filter coupling coefficient to the single mode fibre. Other suitable ultra-narrow band filters such as atomic line and Fabry-Perot etalons [18] can reach $100 \mathrm{pm}$, but are high cost and sensitive to mis-alignment. Hence a narrow band interference filter of $800 \mathrm{pm}$ Full Width Half Maximum (FWHM) bandwidth is preferable [19].

Another method to reduce ambient light level is to reduce the receiver field-of-view (FOV) and, in turn, the transmitted beam divergence. For the $1.57 \mu \mathrm{m}$ sounding wavelength, the diffraction limited beam divergence would be around a few $\mu \mathrm{rad}$. However, atmospheric turbulence causes refractive index variations as seen by the beam front, which leads to phase fluctuations. This gives rise to the wander phenomenon [20]. Within the reviewed literatures, the narrowest FOV designed for the space-borne IPDA application is $50 \mu \mathrm{rad}$ [3].

In terms of detector technology, CHARM-F uses off-theshelf linear InGaAs APD. With the limited avalanche gain associated with InGaAs APDs, the weak received signal photo current is electronically amplified, resulting in a large amount of detector noise. Other emerging technologies such as Mercury Cadmium Telluride ( $\mathrm{HgCdTe}$ ) APDs can achieve a few fW Hz${ }^{-0.5}$ [21]. However, such detectors require cryogenic cooling for low noise operations, a requirement

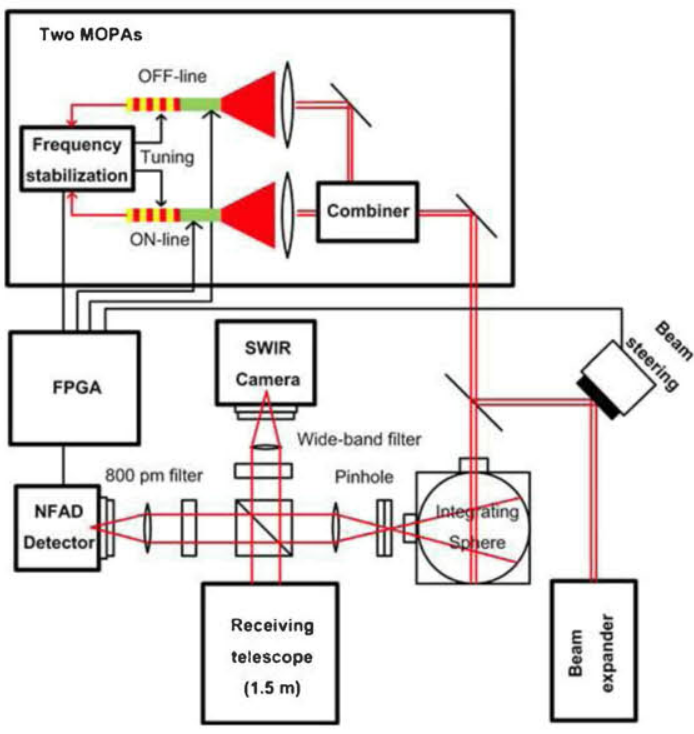

Figure 3. Schematic of the PRSPC IPDA lidar.

which is not ideal in space-borne applications. In comparison to linear mode APDs, SPAD detectors are generally much quieter thanks to the higher Geiger mode gain. In terms of suitable SPAD detector technologies at the targeted wavelength, Princeton Lightwave has recently commercialized InGaAs Negative Feedback Avalanche Photodiode (NFAD). Its dark count rate can be as low as a few kilo counts per second

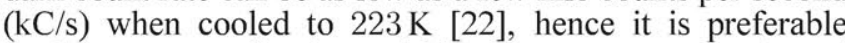
in the target application. Although current generation of NFADs are limited to a $10 \mathrm{MHz}$ saturation count rate (limited due to after-pulses suppression), and the targeted application requires close to $100 \mathrm{MHz}$, detector array [22] that integrates several detector units would provide a feasible solution. With the use of a single photon detector, we refer the proposed lidar instrument as PRSPC IPDA lidar.

\section{PRSPC IPDA lidar Design}

The block diagram of the proposed space-borne PRSPC IPDA lidar is illustrated in Figure 3. The lasers for the on/off line channels are multi-section integrated MOPAs with each containing a DFB section, a modulator section and a tapered amplifier. Three separate electrical contacts will access the three sections on the laser chip: the DFB current controlling the laser emission frequency, the modulation current allows for intensity modulation and generation of the PRBS and the taper current for providing the signal amplification. An optical-electrical feedback loop based on a gas cell reference will be used in each laser chip in order to have highly stable emission frequencies and narrow line-widths. The modulator works either as an optical preamplifier switched on and off or as an electro absorption modulator in reverse bias. The tapered amplifier includes different sections to increase the saturation power and beam quality.

The combined beam is then transmitted through a beam expander with a divergence of $50 \mu \mathrm{rad}$. The modulation bit rate is set to 25 megabits per second $(\mathrm{Mb} / \mathrm{s}$ ) (clock period of $40 \mathrm{~ns}$ ), which provides a distance resolution of $6 \mathrm{~m}$. The bandwidth of the modulation signal can be constrained below $25 \mathrm{MHz}$ with a digital low-pass filter and a digital-to-analog converter to avoid further spectrum broadening, although such broadening effects can be measured and subsequently canceled by applying the appropriate effective absorption 
cross-section.

The backscattered light (referred as the received on/off line channels) is collected by a reflective telescope with a FOV matching the laser beam divergence. The collimated light then passes through a narrow band interference filter with $800 \mu \mathrm{m}$ FWHM bandwidth, before being focused onto a NFAD detector. As shown in Eq. (4), the measurement of the Differencial Absorption Optical Depth (DAOD) involves not only the on/off line energy of the returned channels but also the accurate measurement of the on/off line transmitted energy via the transmitted channels. Such measurements can be taken from sampling the combined beams via mirror backleakage and an integrating sphere (for the purpose of homogenizing and attenuation). A Short Wave Infrared (SWIR) camera is used for alignment.

At the NFAD detector, an incident photon triggers an avalanche event, which outputs an electrical pulse. These pulses are then digitized as time tags by a Time-to-Digital Converter (TDC) implemented in a Field Programmable Gate Array (FPGA). The reference random pulse train signal is synchronized to the transmitted PRBS, and is also digitized by the TDC. Correlation of these time tags provides results that are equivalent to those obtained in a pulsed system. In the obtained correlaiton results, the last return bins record the $N_{o n, o f f}^{r}$ and their positions indicate the distance to the ground surface, and the transmitted channels as $N_{o n, o f f}^{t}$. All digital domain signal processing and the multichannel TDC can be implemented in a singular radiation hardened FPGA.

Other system parameters and operating conditions are shown in Table 1, in comparison with an equivalent pulsed system. The $\mathrm{CO}_{2}$ retrieval precision of the proposed system is 1.50 $\mathrm{ppm}(0.553 \mathrm{ppm}$ for the pulsed system). Although this value is at the boundary of future IPDA mission specifications, it is a worst case scenario due to the surface reflectivity used in the calculation referring to that of the ocean's (which has the lowest surface albedo). For measurements taken across vegetation, the $\mathrm{CO}_{2}$ retrieval precision is improved to 0.773 $\mathrm{ppm}$. These results are limited by the ambient light level, so if a lower bandwidth filter could be applied, a better precision would be achieved. Another major limitation of the PRSPC system is the low detection efficiency offered by present day detectors. If the detection efficiency could be increased to $60 \%$, a typical figure for existing commercially available silicon SPADs, higher precision would be achievable.

\section{Preliminary Experimental Results}

To demonstrate the PRSPC and the feasibility of the NFAD detector in space-borne applications, we have adapted the Qumet MT-30A [23] single photon time tagger that is currently being commercialized by Bristol University to include a real-time multi-channel single photon correlator. The MT30A contains a SPARTAN 6 FPGA in its core and fast signal conditioning peripherals (digitally controlled discriminators and delay-lines) for up to 8 independent channels.

The experimental setup is illustrated in Figure 4. The aims of experiments are to verify that the measured ground return energy would be proportional to the change of the on-line atmospheric transmittance and the ground albedo can be canceled out by the differential measurements. In the setup a $10 \mathrm{~mW}$ CW $1.57 \mu \mathrm{m}$ tunable DFB laser is used as the source, and it is coupled into an optical isolator and is then linked to the Acousto-optic Modulator (AOM) (modulated by

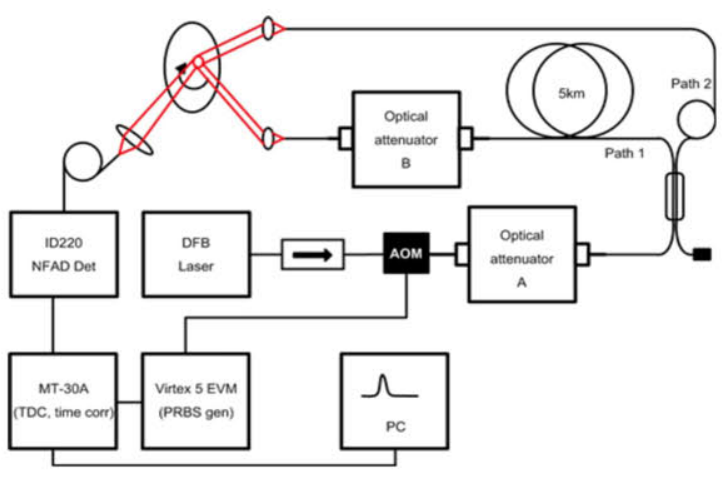

Figure 4. Preliminary experiment setup.

$10 \mathrm{MHz}$ PRBS) via a single mode fiber. Following which, light is attenuated by variable optical attenuator A, which simulates the ground albedo changes. The attenuated light is then split into two paths (1 and 2) via a 1:2 fiber splitter. Path 1 is connected to a $5 \mathrm{~km}$ fiber to mimic the ground-tosatellite distance and subsequently, another variable optical attenuator B emulates the effect of differences in on-line atmospheric transmittances. Subsequently, the light exits the optical attenuator B and is projected onto a rotating diffusive surface (white tape, disc and a DC motor). This is to reduce laser speckle noise and to simulate the ground's motion in reference to the orbiting satellite. Light from the path 2 directly illuminates the rotating diffusive target, simulating the transmitted on-line channel.

The reflected light, a combination of both path 1 and 2 is then collected by an off-the-shelf InGaAs NFAD based single photon detector module (IDQuantique id220) via a $60 \mu \mathrm{m}$ multi-mode fiber. The output of the NFAD detector is then connected to the MT-30A. The other two reference PRBS triggers are generated by the Virtex 5 FPGA evaluation module, which synchronizes the MT-30a's correlation process to the PRBS triggers. This allows for three trigger channels to be time tagged and correlated in real-time.

Each correlation waveform is a histogram with 10 second
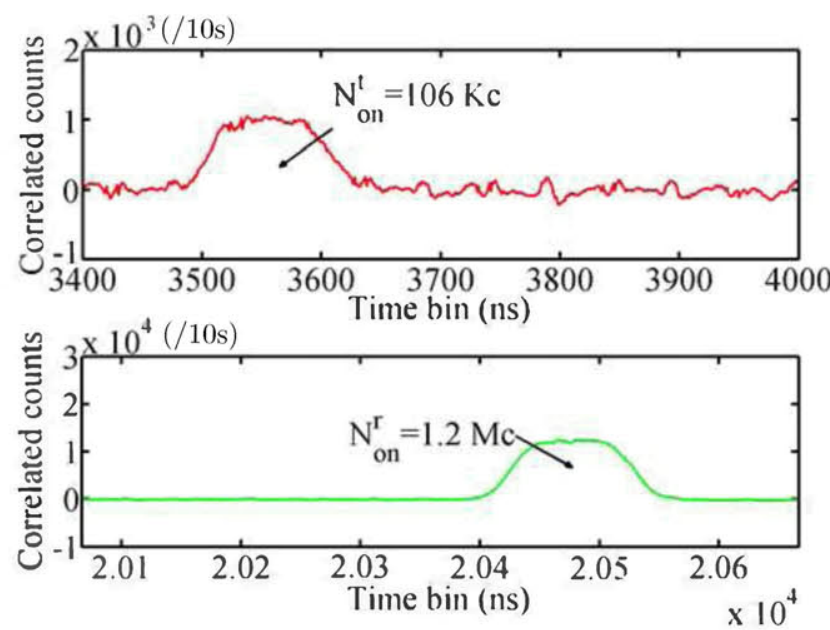

Figure 5. Correlation results, with $10 \mathrm{~s}$ integration time and 1 ns per bin: red) the transmitted on-line channel; green) the returned on-line channel, each pulse is about $100 \mathrm{~ns}$ wide. 
integration time. Examples of correlation waveforms around the returned pulses are plotted in Figure 5, showing results which could be obtained by a pulsed system without any distortions. By integrating the photoelectron counts in the two pulses, photoelectrons numbers indicating the ground return energy $N_{o n}^{r}$, and photoelectrons numbers indicating the transmitted signal energy $N_{o n}^{t}$ can be both obtained.

\section{Linearity Experiment}

The measurement linearity of the returned photoelectron number per correlation ( $10 \mathrm{~s})$ to the applied attenuation level in the optical attenuator B simulates the linearity of the transmittance measurements in space-borne remote sensing scenarios. Measurement results are shown in Figure 6. In which, the optical attenuator B has been swept from $5 \mathrm{~dB}$ $(2.5 \mathrm{Mc}$ per $10 \mathrm{~s})$ to $30 \mathrm{~dB}(1 \mathrm{Kc}$ per $10 \mathrm{~s})$ in steps of $0.01 \mathrm{~dB}$ per integration time $(10 \mathrm{~s})$. The green line shows the linearity plot of the received on-line channel count vs attenuation, and the red line is the linearity plot of the fixed level transmitted on-line channel. First impressions may lead to the assumption that the single photon counting techniques are highly non-linear for count rates higher than $100 \mathrm{Kc}$ per $10 \mathrm{~s}$, which is mainly caused by the $1 \mu \mathrm{s}$ dead time of the detector. Such non-linearity cannot be easily calibrated out because the 'missing counts' caused by the dead-time are proportional to the signal correlated count rate as well as the total count rate.

Fortunately, in an IPDA system, the absolute energy of the ratio of $N_{o n}^{r}$ to $N_{o f f}^{r}$ is of concern. This ratio is plotted in blue in Figure 6, demonstrating a very high linearity relationship up to $1 \mathrm{Mc}$ per $10 \mathrm{~s}$. This is due to the non-linearity cancellation of two correlated signals detected from the same detector, with the same total count rate. The theoretical linear section of the blue trace should be even higher to almost 5 Mc per $10 \mathrm{~s}$, the deviation from the experiment and theoretical values are suspected to be caused by another non-linear effect in single photon correlation experiments, namely the pileup effect [24], where the detected photoelectrons count rate becomes higher than $1 \%$ of the pulse transmission rate $(1 \mathrm{Mc}$ per $10 \mathrm{~s}$ vs $10 \mathrm{Mc} / \mathrm{s}$ in our case).

Note that the $1 \mathrm{MHz}$ saturation count rate of the id220 is caused by the minimum dead-time of $1 \mu \mathrm{s}$. This is to guarantee the after-pulse probabilities can be constrained to an

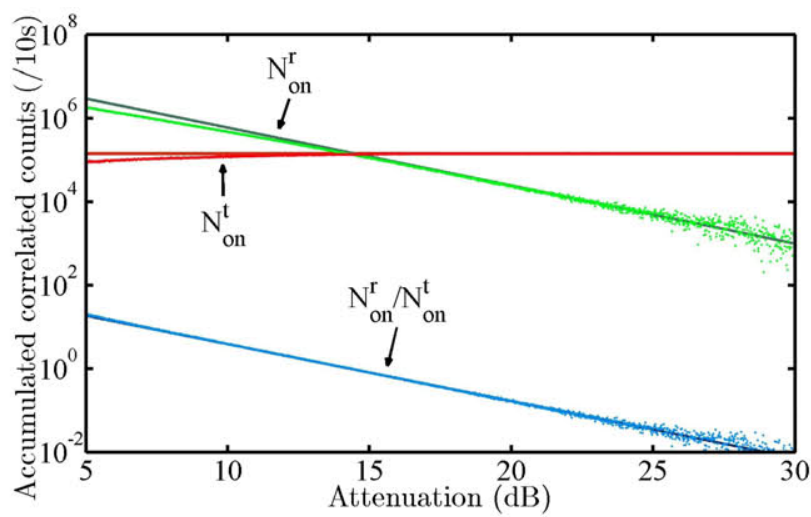

Figure 6. Linearity experiment results, with each data point (lighter colors) representing accumulated correlated counts during $10 \mathrm{~s}$ integration and solid lines (darker colors) are obtained from fittings to the $15-25 \mathrm{~dB}$ most linear sections. acceptable level. Future firmware updates may allow for a higher saturation count rate. In such a situation, afterpulsing probability can be minimized by raising the operating temperature, at the expense of increasing the dark count rate from a few Kc/s to $100 \mathrm{Kc} / \mathrm{s}$. Such modifications will make the id220 suitable for space-borne IPDA applications which require a count rate of tens of $\mathrm{MHz}$, whilst still being orders of magnitude quieter than linear mode devices. The worst case implementation is the use of multiple detectors sharing the counting load.

\section{Surface Albedo and Precision Experiment}

In space-borne lidar, the ground albedo and aerosol attenuation encountered by the on/off line returned channels should be nearly identical. Hence, the differential absorption measurements are insensitive to these parameters in contrast to absolute absorption measurements. To verify this property, we consider that path 1 and path 2 represent received on-line and off-line channels respectively, and the $5 \mathrm{~km}$ delay is to simulate the code delay of half the PRBS repetition period. In this experiment, variable optical attenuator B is set to a fixed value, and a variable optical attenuator A is randomly varied to simulate ground albedo changes encountered by the satellite. The results are shown in Figure 7, in which the light and dark green curves represent the absolute values obtained from the emulated on/off lines, showing fluctuations with respect to the setting of the attenuator $\mathrm{A}$. However, their ratio shown in brown is almost invariant through this experiment with a mean value of 3.34 and standard deviation of 0.025 . Small fluctuations of this ratio are caused by signal shot noise. We can now translate these fluctuations to precision of obtained DOAD via Eq. (4), then the XCO2 retrieval precision by Eq. (5). Assuming an accurate knowledge of the weighting function (denominator of Eq. (5)) of the value 0.0015 , and a non-biased measurement of the transmitted on/off line energy ratio of 1 . The averaged XCO2 is 400 ppm and the expected $\mathrm{XCO} 2$ retrieval precision under these received on/off-line count rates is $2.8 \mathrm{ppm}$. Note that, this experiment does not consider the shot noise from ambient light. However, it provides enough evidence that if the saturation count rate of the detector can be improved to $100 \mathrm{Mc} / \mathrm{s}$, such that a nominal linear count of $10 \mathrm{Mc} / \mathrm{s}$ can be achieved, single photon counting approaches are highly applicatable to space-borne scenarios. The random errors (precision) would be improved to meet the specification. The measurement stability will be evaluated thoroughly in future experimental setups.

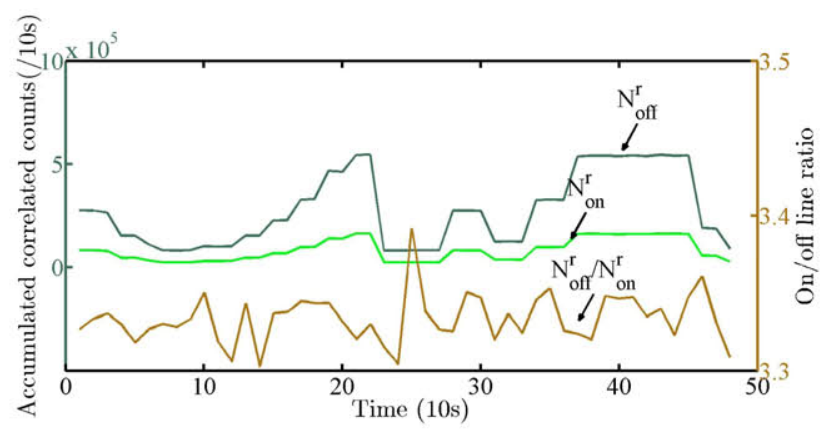

Figure 7. Ground albedo variation simulation results, with manually varied attenuation, the on/ off line return channels are recoded for 10 s integration and shown in light and dark green curves; their ratio is shown in brown, with mean of 3.34 and s.d. of 0.025 . 


\section{SUMMARY}

Future space missions aim to accurately measure the $\mathrm{CO}_{2}$ concentration utilizing space-borne IPDA lidars. In this paper, the results of a feasibility study of the application of PRN modulation in such systems have been presented. In comparison to the sequential transmissions of the on-line and off-line wavelengths experienced in existing pulsed systems, the proposed instrument transmits both wavelengths simultaneously. Hence, the laser footprint misalignment problem can be eliminated. Furthermore, with $\mathrm{CW}$ sources as an alternative to pulsed ones, the proposed system can be more robust and should attain better wall-plug efficiency. When compared with existing sine wave modulated CW IPDA lidar concepts, PRN modulation eliminates the interference from unwanted backscattering from circus clouds and dust. The envelope SNR of the proposed system has been analyzed in comparison to the pulsed system. Based on this analysis, optimized system parameters and setup arrangements are given in light of the existing technology. Although the proposed scheme may be more susceptible to ambient and detector noise, it has shown that a worst case retrieval precision of $1.5 \mathrm{ppm}$ over $50 \mathrm{~km}$ path integration can be achieved theoretically. Preliminary experiments have proven the feasibility of single photon detection and time correlated counting. It is also to be expected that the precision will be further improved with newer detection and receiver technologies.

\section{ACKNOWLEDGMENTS}

This work was supported by the European Commission through the project BRITESPACE under grant agreement no. 313200 . We also would like to thank IDQuantique (IDQ) for loaning us the id 220 detector module.

\section{REFERENCES}

[1] G. Ehret, C. Kiemle, M. Wirth, A. Amediek, A. Fix, and S. Houweling, "Space-borne remote sensing of $\mathrm{CO}_{2}$, $\mathrm{CH}_{4}$, and $\mathrm{N}_{2} \mathrm{O}$ by integrated path differential absorption lidar: a sensitivity analysis," Applied Physics B: Lasers and Optics, vol. 90, no. 3, pp. 593-608, 2008.

[2] M. Quatrevalet, et al. "CHARM-F: The airborne integral path differential absorption lidar for simultaneous measurements of atmospheric $\mathrm{CO}_{2}$ and $\mathrm{CH}_{4}$. in Proc. 25th Int. Laser Radar Conf., St. Peterburg, Russia, vol.1, 2010.

[3] S. Kameyama, et al., "Feasibility study on $1.6 \mu \mathrm{m}$ continuous-wave modulation laser absorption spectrometer system for measurement of global $\mathrm{CO}_{2}$ concentration from a satellite," Applied optics, 2011. 50(14): p. 20552068.

[4] N. Takeuchi, N. Sugimoto, H. Baba, and K. Sakurai, "Random modulation cw lidar," Applied optics, vol. 22, no. 9, pp. 1382-1386, 1983.

[5] C. Nagasawa, M. Abo, H. Yamamoto, and O. Uchino, "Random modulation $\mathrm{cw}$ lidar using new random sequence," Applied optics, vol. 29, no. 10, pp.1466-1470, 1990.

[6] X. Ai, R. Nock, N. Dahnoun, and J.G. Rarity, "Highresolution random-modulation cw lidar," Applied Optics, vol. 50, no. 22, pp. 4478-4488, 2011.

[7] V. Mitev, R. Matthey, and D. Reusser, "Development of a pseudorandom noise modulation, continuous-wave
(PRN-cw) total backscatter lidar," in Proceedings of SPIE, vol. 2505 , 1995 , p. $150-160$.

[8] B. L. Campbell, J.F. and A. Nehrir, "Advanced sine wave modulation of continuous wave laser system for atmospheric $\mathrm{CO}_{2}$ differential absorption measurements," arXiv preprint arXiv:1309.3542, 2013.

[9] E. Browell, S. Ismail, and W. Grant, "Differential absorption lidar (DIAL) measurements from air and space," Applied Physics B, vol. 67, no. 4, pp. 399-410, 1998.

[10] A. Fix, G. Ehret, J. Lohring, D. Hoffmann, and M. Alpers, "Water vapor differential absorption lidar measurements using a diode-pumped all-solid-state laser at 935 nm," Applied Physics B, vol. 102, no. 4, pp. 905-915, 2011.

[11] K. Numata, J. R. Chen, S. T. Wu, J. B. Abshire, and M. A. Krainak, "Frequency stabilization of distributedfeedback laser diodes at $1572 \mathrm{~nm}$ for lidar measurements of atmospheric carbon dioxide," Applied Optics, vol. 50, no. 7 , pp. 1047-1056, 2011.

[12] B. P. Fox, K. Simmons-Potter, W. Thomes, and D. A. Kliner, "Gamma-radiation-induced photodarkening in unpumped optical fibers doped with rare-earth constituents. Nuclear Science," IEEE Transactions on, vol. 57, no. 3, pp. 1618-1625, 2010.

[13] BRITESPACE Consortium, "High Brightness Semiconductor Laser Sources for Space Applications in Earth Observation (BRITESPACE)," EU FP7 project no. 313200. URL:http://www.britespace.eu/, last retrieved: Nov. 15th, 2013.

[14] A. McCarthy, et al., "Long-range time-of-flight scanning sensor based on high-speed time-correlated singlephoton counting," Applied optics, vol. 48, no. 32, pp. 6241-6251, 2009.

[15] Q. Zhang, L. Chen, and N. Chen, "Pseudo-random single photon counting: a high-speed implementation," Biomedical optics express, vol. 1, no. 1, pp. 41-46, 2010.

[16] J. Caron and Y. Durand, "Operating wavelengths optimization for a spaceborne lidar measuring atmospheric $\mathrm{CO}_{2}$," Applied optics, vol. 48, no. 28, pp. 5413-5422, 2009.

[17] R. De Young, "A narrow band fiber Bragg grating filter for lidar receivers," in Lasers and Electro-Optics, 2001. CLEO'01. Technical Digest. Summaries of papers presented at the Conference on. IEEE, 2001, pp. 494-495.

[18] J. Lumeau, V. Smirnov, A. Glebov, and L. B. Glebov, "Ultra-narrow bandpass filters based on volume Bragg grating technologies," in SPIE Defense, Security, and Sensing, International Society for Optics and Photonics, $2010,76750 \mathrm{H}$.

[19] J. Abshire, H. Riris, G. Allan, C. Weaver, J. Mao, X. Sun, W. Hasselbrack, S. Kawa, and S. Biraud, "Pulsed airborne lidar measurements of atmospheric $\mathrm{CO}_{2}$ column absorption," Tellus B, vol. 62, no. 5, pp. 770-783, 2010.

[20] Y. Cheon, Angle-of-arrival fuctuations of optical waves in the atmospheric surface layer, 2008.

[21] K. Foubert, et al. "Development of $\mathrm{HgCdTe}$ singleelement APDs based detectors for low flux short wave infrared applications," in SPIE OPTO. International Society for Optics and Photonics, 2013, 86210F.

[22] X. Jiang, et al. "InGaAs/InP negative feedback avalanche diodes (NFADs)," in SPIE Defense, Security, 
and Sensing, International Society for Optics and Photonics, 2011.

[23] R. Nock, Qumet technology, [cited 2013 15/11]; MT-30A flexible instrument platform]. Available from: http://www.qumetec.com/

[24] W. Becker, A. Bergmann, G. Biscotti, K. Koenig, I. Riemann, L. Kelbauskas, and C. Biskup, "High-speed FLIM data acquisition by time-correlated single-photon counting," in Biomedical Optics 2004. International Society for Optics and Photonics, 2004, pp. 27-35.

\section{BIOGRAPHY}

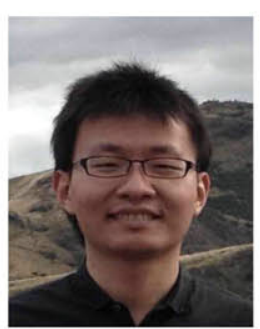

Xiao $\mathrm{Ai}$ is a postdoctoral researcher at Bristol University, having recently graduated from his PhD program which specialized in $3 D$ imaging techniques. Xiao's research involves the development of lidar technologies: for atmospheric sensing and automotive obstacle detection. Xiao also has extensive experience on optoelectronic systems, analogue and digital signal processing and

machine vision.

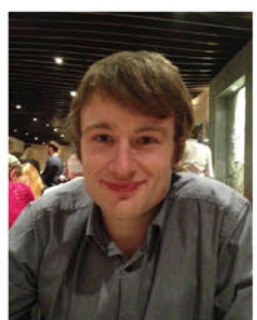

Richard William Nock is completing PhD student at Bristol University. He completed his MSc degree at Bristol in 2007, before working in industry prior to entering the PhD program. He has been developing technologies to make low cost timing instruments based on FPGA technology for quantum information applications. Research has focused on time to digital conversion, low cost electronics for Quantum Key Distribution and short pulse LED driving.

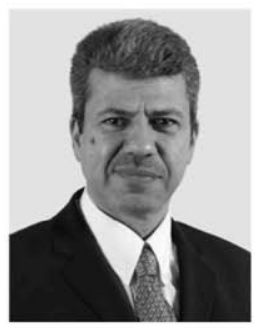

Naim Dahnoun Reader in the Electrical and Electronic Engineering at the University of Bristol. He obtained his $P h D$ in Biomedical Engineering in 1990 and is the Programme Director for Computer Science and Electronics at the University of Bristol. Naim has over 25 years experience in real-time DSP and, in recognition of the important role played by Universities in educating engineers in new technologies such as real-time Digital Signal Processing (DSP).Naim's main research interests include Real-Time Digital Signal Processing applied to Biomedical Engineering, Video Surveillance, Automotive and Optics.

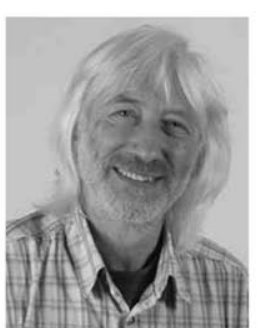

John Rarity is the head of the Photonics group in Electrical Engineering at University of Bristol and has recently been awarded an ERC Advanced Grant. His developments of experimental quantum interference and quantum cryptography earned him the Thomas Young medal from the IOP in 1995 and established him as a globally recognized leader in quantum optics. Prof. Rarity has participated in, and coordinated, European research projects continuously for the last 18 years (including Descartes prize winner in 2004 (Qucomm)), and published over 100 journal papers (h-factor 34 ).

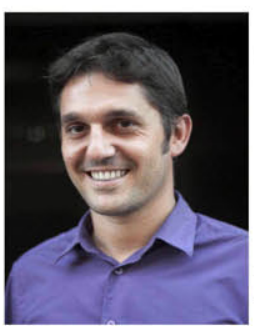

Antonio Consoli received his $M S c$ degree in Electronic Engineering from Universitá degli Studi di Pavia, Italy, in 2004 with specialization in optoelectronics. Since 2007 he is at Universidad Politécnica de Madrid, Spain, where he obtained his PhD degree in 2011. He is currently working as post-doc researcher and his main interests are in semiconductor laser dynamics, timefrequency analysis, pulse shaping, pulse characterization and high brightness semiconductor lasers.

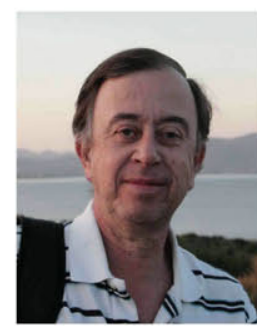

Ignacio Esquivias (Madrid, 1955) received the M.Sc. and Ph.D. degrees in Electronic Engineering from the Universidad Politécnica de Madrid in 1977 and 1983, respectively, becoming Full Professor at this university in 2001. He leads the Laser Diode Group of the CEMDATIC, with activity in laser diode modeling and characterization since 1992. He has been PI in 10 national and 5 EC funded international projects on laser diodes, and he has published more than 100 journal and conference papers on this field. He coordinates the EC funded project BRITESPACE.

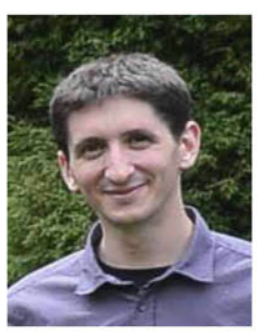

Mathieu Quatrevalet Optical Engineer, Master: He operated groundbased DIAL systems in Antarctica and during an employment at ESA he worked within the A-SCOPE project for $\mathrm{CO}_{2}$. As a research scientist at the lidar Department of DLR Institut für Physik der Atmosphäre. He is involved in the development of the airborne greenhouse gas LIDAR CHARM-F for $\mathrm{CO}_{2}$ and $\mathrm{CH}_{4}$.

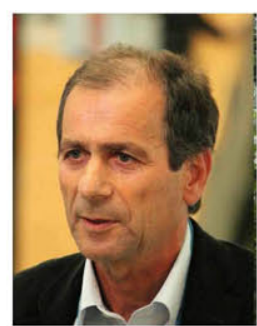

Gerhard Ehret Physicist, Ph.D. in laser spectroscopy, Head of LIDAR department at IPA ( 28 persons), expert in LIDAR instrument development for airborne and space-borne LIDAR missions on water vapor, ozone, $\mathrm{CO}_{2}$, and $\mathrm{CH}_{4}$, long term experience in the development of high power laser systems, analogue detection and operation of LIDAR instruments on airborne platforms. Member of related Mission Advisory Groups (MAG) at ESA (WALESMAG, A-SCOPE-MAG, and MERLIN-SAG) and CoPI of the Franco-German Climate Mission MERLIN. 
Table 1. Envelop SNR estimation for pulsed and PRSPC space-borne IPDA lidars.

Pulsed [1]

Transmitter

On-line pulse energy:

Off-line pulse energy:

Pulse duration (rep rate):

Integration time (int pulses):

Bean divergence (full):

On-line wavelength:

Off-line wavelength:

Receiver

Type:

Area:

Bandpass filter FWHM:

Optical efficiency:

FOV (full):

Detector

Type:

Quantum efficiency:

Excess noise factor:

Noise Equivalent Power:

Misc.

Orbit type:

Solar Zenith angle:

Nadir radiance (ocean-veg):

$L_{r}=1.7-5 \mathrm{~mW} \mathrm{~m}^{-2} \mathrm{sr}^{-1} \mathrm{~nm}^{-1}$

Orbit altitude:

Velocity:

$R=450 \mathrm{~km}$

Surface albedo (ocean-veg):

On/off-line transmittance:

DAOD:

$\mathrm{XCO} 2$ :

Calculated values

Energy to photoelectron coeff: $\xi_{e}=\frac{\eta_{q} \lambda_{o n}}{F_{d} h c}=1.47 \mathrm{e} 18$

On-line photoelectrons accumulated (ocean-veg):

$$
N_{\text {on }}=E_{\text {on }}^{\text {pul }} \cdot \eta_{o} \cdot \alpha_{s} \cdot T_{\text {on }} \cdot \frac{A_{r}}{R^{2}} \cdot \xi_{e}=1520-5060
$$

Off-line photoelectrons per pulse (ocean-veg):

$$
N_{o f f}=E_{o f f}^{p u l} \cdot \eta_{o} \cdot \alpha_{s} \cdot T_{o f f} \cdot \frac{A_{r}}{R^{2}} \cdot \xi_{e}=4850-16200
$$

Ambient photoelectrons per range gate (ocean-veg):

$$
\begin{aligned}
N_{a m b} & =T_{p u l} \cdot \eta_{0} \cdot \Delta_{\lambda} \cdot L_{r} \cdot \pi\left(\frac{\theta_{F O V} \cdot R}{2}\right)^{2} \cdot \frac{A_{r}}{R^{2}} \cdot \xi_{e} \\
& =29.4-86.6
\end{aligned}
$$

Detector dark photoelectrons per range gate:

$$
N_{\text {det }}=\frac{T_{\text {pul }}}{2} \cdot\left(\xi_{e} \cdot N E P_{\text {det }}\right)^{2}=46.7
$$

On-line SNR (ocean-veg):

$$
S N R_{o n}^{p u l}=\frac{N_{o n}}{\sqrt{N_{o n}+N_{a m b}+N_{d e t}}}=38.0-70.2
$$

Off-line SNR (ocean-veg):

$$
S N R_{\text {off }}^{\text {pul }}=\frac{N_{\text {off }}}{\sqrt{N_{\text {off }}+N_{a m b}+N_{\text {det }}}}=69.1-127
$$

$\mathrm{XCO} 2$ precision (ocean-veg):

$$
\begin{aligned}
\sigma_{x c o 2} & =\frac{X C O 2}{2 \cdot D A O D} \cdot \sqrt{\frac{\left(S N R_{o n}^{p u l}\right)^{-2}+\left(S N R_{o n}^{p u l}\right)^{-2}}{M}} \\
& =0.553-0.3 \mathrm{ppm}
\end{aligned}
$$

\section{Proposed PRSPC}

Transmitter

$$
\begin{array}{ll}
\text { On-line average power: } & P_{\text {on }}^{a v g}=2 \mathrm{~W} \\
\text { Off-line average power: } & P_{o f f}^{a v g}=2 \mathrm{~W} \\
\text { Chip (bit) time: } & T_{c}=40 \mathrm{~ns} \\
\text { Integration time: } & T_{\text {int }}=7 \mathrm{~s} \\
\text { Bean divergence (full): } & \theta_{D I}=50 \mu \mathrm{rad} \\
\text { On-line wavelength: } & \lambda_{\text {on }}=1572.02 \mathrm{~nm} \\
\text { Off-line wavelength: } & \lambda_{o f f}=1572.11 \mathrm{~nm}
\end{array}
$$

Receiver

Type:

Area:

Bandpass filter FWHM:

Optical efficiency:

FOV (full):

Detector

Type:

Detection efficiency:

Excess noise factor:

Detector dark count:

Cassegrain telescope

$A_{r}=1.77 \mathrm{~m}^{2}$

$\Delta_{\lambda}=800 \mathrm{pm}$

$\eta_{0}=80 \%$

$\theta_{F O V}=50 \mu \mathrm{rad}$

Misc.

Orbit type:

Multiple NFADs

$\eta_{e}=30 \%$

$F_{d}=1$

$N_{d c}=500 \mathrm{Kc} / \mathrm{s}$

Solar Zenith angle:

6-h sun synchronous

$\theta_{z e n}=75^{\circ}$

Off-line SNR (ocean-veg):

$$
L_{r}=1.7-5 \mathrm{~mW} \mathrm{~m}^{-2} \mathrm{sr}^{-1} \mathrm{~nm}^{-1}
$$

Orbit altitude:

$R=450 \mathrm{~km}$

Velocity:

$v=7 \mathrm{~km} \mathrm{~s}^{-1}$

Surface albedo (ocean-veg): $\quad \alpha_{s}=0.03-0.1 \mathrm{sr}^{-1}$

On/off-line transmittance: $\quad T_{\text {on }}=0.3, T_{\text {off }}=0.78$

DAOD:

$D A O D=0.48$

$\mathrm{XCO} 2$ :

$X C O 2=400 \mathrm{ppm}$

\section{Calculated values}

Energy to photoelectron coeff: $\xi_{e}=\frac{\eta_{e} \lambda_{o n}}{F_{d} h c}=2.37 \mathrm{e} 18$

On-line photoelectrons accumulated (ocean-veg):

$N_{\text {on }}=P_{\text {on }}^{a v g} \cdot T_{\text {int }} \cdot \eta_{o} \cdot \alpha_{s} \cdot T_{\text {on }} \cdot \frac{A_{r}}{R^{2}} \cdot \xi_{e}=3.20-10.5 \mathrm{M}$

Off-line photoelectrons per pulse (ocean-veg):

$N_{\text {off }}=P_{o f f}^{a v g} \cdot T_{\text {int }} \cdot \eta_{o} \cdot \alpha_{s} \cdot T_{\text {off }} \cdot \frac{A_{r}}{R^{2}} \cdot \xi_{e}=8.2-27.3 \mathrm{M}$

Ambient photoelectrons accumulated (ocean-veg):

$$
\begin{gathered}
m \cdot N_{a m b}=m \cdot T_{c} \cdot \eta_{0} \cdot \Delta_{\lambda} \cdot L_{r} \cdot \pi\left(\frac{\theta_{F O V} \cdot R}{2}\right)^{2} \cdot \frac{A_{r}}{R^{2}} \cdot \xi_{e} \\
=42.5-125 \mathrm{M}
\end{gathered}
$$

Detector equivalent photoelectrons:

$$
m \cdot N_{d e t}=m \cdot T_{c} \cdot N_{d c}=1.75 \mathrm{M}
$$

On-line SNR (ocean-veg):

$$
S N R_{o n}^{p r n}=\frac{N_{o n}}{\sqrt{2} \sqrt{N_{o n}+N_{o f f}+m \cdot N_{a m b}+m \cdot N_{d e t}}}=298-577
$$

Off-line SNR (ocean-veg):

$$
S N R_{o f f}^{p r n}=\frac{N_{o f f}}{\sqrt{2} \sqrt{N_{o f f}+N_{o n}+m \cdot N_{a m b}+m \cdot N_{\text {det }}}}=778-1510
$$

$\mathrm{XCO} 2$ precision (ocean-veg):

$$
\begin{aligned}
\sigma_{x c o 2} & =\frac{X C O 2}{2 \cdot D A O D} \cdot \sqrt{\left(S N R_{o n}^{p r n}\right)^{-2}+\left(S N R_{o n}^{p r n}\right)^{-2}} \\
& =1.50-0.773 \mathrm{ppm}
\end{aligned}
$$

